\title{
A cubic B-spline Galerkin approach for the numerical simulation of the GEW equation
}

\author{
S. Battal Gazi Karakoç ${ }^{1}$, Halil Zeybek ${ }^{2, *}$ \\ ${ }^{1}$ Department of Mathematics, Faculty of Science and Art, Nevsehir Haci Bektas Veli University, 50300 Nevsehir, Turkey. \\ ${ }^{2}$ Department of Applied Mathematics, Faculty of Computer Science, Abdullah Gul University, 38080 Kayseri, Turkey.
}

(Received: 11 September 2015; Accepted: 03 December 2015)

\begin{abstract}
The generalized equal width (GEW) wave equation is solved numerically by using lumped Galerkin approach with cubic B-spline functions. The proposed numerical scheme is tested by applying two test problems including single solitary wave and interaction of two solitary waves. In order to determine the performance of the algorithm, the error norms $L_{2}$ and $L_{\infty}$ and the invariants $I_{1}, I_{2}$ and $I_{3}$ are calculated. For the linear stability analysis of the numerical algorithm, von Neumann approach is used. As a result, the obtained findings show that the presented numerical scheme is preferable to some recent numerical methods.
\end{abstract}

Keywords Galerkin Method, Finite Element Method, GEW Equation, Cubic B-spline, Solitary Waves

AMS 2010 subject classifications 41A15, 65N30, 76B25

DOI: $10.19139 /$ soic.v4i1.167

\section{Introduction}

Firstly, Peregrine [1] presented the regularized long wave (RLW) equation derived from long waves propagating in the positive $x$-direction as a model for small-amplitude long waves on the surface of water in a channel. Later, Korteweg-de Vries (KdV) equation, which describes the long waves in non-linear dispersive systems, was introduced by Benjamin et al. [2]. The equal width (EW) wave equation was used by Morrison et al. [3] as an alternative model to the RLW and the KdV equations. The EW equation is obtained by taking $p=1$ in the generalized equal width (GEW) wave equation. So, the GEW equation is based upon the EW equation and is related to the generalized regularized long wave (GRLW) equation and the generalized Korteweg-de Vries $(\mathrm{GKdV})$ equation. These general equations are nonlinear wave equations with $(p+1)$ th nonlinearity and have solitary solutions, which are pulse-like Raslan [7]. The GEW equation is given by the following form:

$$
U_{t}+\varepsilon U^{p} U_{x}-\mu U_{x x t}=0,
$$

with the boundary and initial conditions

$$
\begin{aligned}
& U(a, t)=0, \quad U(b, t)=0, \\
& U_{x}(a, t)=0, \quad U_{x}(b, t)=0, \\
& U(x, 0)=f(x), \quad a \leq x \leq b,
\end{aligned}
$$

\footnotetext{
*Correspondence to: Halil Zeybek (Email: halil.zeybek@agu.edu.tr). Department of Applied Mathematics, Faculty of Computer Science, Abdullah Gul University, 38080 Kayseri, Turkey.
}

ISSN 2310-5070 (online) ISSN 2311-004X (print)

Copyright (C) 2016 International Academic Press 
and physical boundary conditions $U \rightarrow 0$ as $x \rightarrow \pm \infty$, where the subscripts $t$ and $x$ represent spatial and time differentiation, $p$ is a positive integer, $\varepsilon$ and $\mu$ positive constant. $f(x)$ is a localized disturbance inside the interval $[a, b]$ and it will be determined later. In the fluid problems, $U$ is related to the wave amplitude of the water surface or similar physical quantity. In the plasma applications, $U$ is negative of the electrostatic potential. Therefore, the solitary wave solution of Eq.(1) has an important role in the motion of non-linear dispersive waves.

Until now, the EW equation has been solved by using many analytical and numerical solution techniques. Galerkin method based on cubic B-spline functions and Petrov-Galerkin method based on quadratic B-spline functions was used by Gardner et al. [11, 12]. Zaki [13] presented a least-squares finite element scheme to obtain the numerical solution of the EW equation. Dogan [14], Esen [15] and Saka [16] obtained the numerical solution of the equation with Galerkin's method using linear finite elements, lumped Galerkin method using quadratic B-splines, space-splitting technique and Galerkin method using quadratic B-spline, respectively.

If $p=2$ in Eq.(1), the obtained equation is known as the modified equal width (MEW) wave equation. The MEW equation has been studied by many researchers. A lumped Galerkin method was set up by Esen [17] with quadratic B-splines. Collocation approach was used by Saka [18] for the numerical solution of the MEW equation. A lumped Galerkin method and Petrov-Galerkin method based on cubic B-splines have been implemented to the MEW equation by Karakoç and Geyikli [19, 20].

In the literature, there are limited number of studies on the GEW equation. The exact solitary wave solutions of the generalized EW and the generalized EW-Burges equation were obtained by Hamdi et al. [5]. Evans and Raslan [6], Raslan [7] presented the collocation method based on quadratic, cubic B-splines to get the numerical solution of the GEW equation. Petrov-Galerkin finite element method using a quadratic B-spline function as the trial function was investigated for solving the GEW equation by Roshan [8]. The GEW equation was solved numerically using the meshless method based on a global collocation with standard types of radial basis functions (RBFs) by Panahipour [9]. Taghizadeh et al. [10] have constructed the homogeneous balance method to obtain the exact travelling wave solutions of the GEW equation.

Galerkin finite element method based on B-spline functions, which is discussed here, has been used to obtain the numerical solution of nonlinear modeling problems by many authors. Gardner and Gardner [21] applied the Galerkin method with cubic B-splines to the RLW equation. Doğan [22] introduced the Galerkin's method using linear space finite elements to obtain the numerical results of the RLW equation. Dağ et al. [23] solved the RLW equation using the quintic B-spline Galerkin approach. Saka and Dă̆ [24], Kutluay and Uçar [25], Karakoç et al. [26] have proposed the Galerkin method based on quartic, quadratic, cubic B-spline functions to acquire the numerical solutions of KdVB, Coupled KdV, MRLW equations, respectively. Lately, numerical solutions of the fractional diffusion and fractional diffusion-wave equations, an Improved Boussinesq type equation, a coupled $\mathrm{mKdV}$ equation have been obtained by means of quadratic B-spline Galerkin scheme [28, 29, 30]. Also, B-splines have been used for applying the collocation method as an approximation function to get the numerical solution of Kawahara equation [27].

When we look at the numerical results of nonlinear modeling problems, the Galerkin approach is an accurate and efficient numerical technique. Besides, because of the second derivative to $x$ in Eq.(1), the approximate function must be at least second order (quadratic, cubic and so on). That's why, in this paper, we have implemented the lumped Galerkin method using cubic B-splines to the GEW equation.

\section{A Lumped Galerkin Method}

Let us consider the solution domain limited to a finite interval $[a, b]$. The interval $[a, b]$ is divided into $N$ equal subinterval by the points $x_{m}$ such that $a=x_{0}<x_{1} \cdots<x_{N}=b$ and length $h=\frac{b-a}{N}=\left(x_{m+1}-x_{m}\right)$. The cubic 
B-spline functions $\phi_{m}(x),(m=-1(1) N+1)$, at the nodes $x_{m}$ which form a basis over the interval $[a, b]$ are described by Prenter [4]

$$
\phi_{m}(x)=\frac{1}{h^{3}} \begin{cases}\left(x-x_{m-2}\right)^{3}, & x \in\left[x_{m-2}, x_{m-1}\right), \\ h^{3}+3 h^{2}\left(x-x_{m-1}\right)+3 h\left(x-x_{m-1}\right)^{2}-3\left(x-x_{m-1}\right)^{3}, & x \in\left[x_{m-1}, x_{m}\right), \\ h^{3}+3 h^{2}\left(x_{m+1}-x\right)+3 h\left(x_{m+1}-x\right)^{2}-3\left(x_{m+1}-x\right)^{3}, & x \in\left[x_{m}, x_{m+1}\right), \\ \left(x_{m+2}-x\right)^{3}, & x \in\left[x_{m+1}, x_{m+2}\right], \\ 0 & \text { otherwise. }\end{cases}
$$

Because each cubic B-spline $\phi_{m}$ covers 4 intervals, each finite interval $\left[x_{m}, x_{m+1}\right]$ is covered by 4 splines. The approximate solution $U_{N}(x, t)$ is written in terms of the cubic B-spline functions as

$$
U_{N}(x, t)=\sum_{j=-1}^{N+1} \phi_{j}(x) \delta_{j}(t),
$$

in which the unknown $\delta_{j}(t)$ are time-dependent quantities and they will be calculated by using the boundary and weighted residual conditions. Using the equality $h \eta=x-x_{m}$ such that $0 \leq \eta \leq 1$, the finite interval $\left[x_{m}, x_{m+1}\right]$ is converted into more easily workable interval $[0,1]$. In this case, the cubic B-splines (3) depending on variable $\eta$ over the gap $[0,1]$ are reconstructed in the following form:

$$
\begin{aligned}
& \phi_{m-1}=(1-\eta)^{3}, \\
& \phi_{m}=1+3(1-\eta)+3(1-\eta)^{2}-3(1-\eta)^{3}, \\
& \phi_{m+1}=1+3 \eta+3 \eta^{2}-3 \eta^{3}, \\
& \phi_{m+2}=\eta^{3} .
\end{aligned}
$$

Here we should state that all cubic B-spline functions except that $\phi_{m-1}(x), \phi_{m}(x), \phi_{m+1}(x)$ and $\phi_{m+2}(x)$ are null over the finite element $[0,1]$. Therefore, approximation function (4) in terms of element parameters $\delta_{m-1}, \delta_{m}, \delta_{m+1}, \delta_{m+2}$ and B-spline element shape functions $\phi_{m-1}, \phi_{m}, \phi_{m+1}, \phi_{m+2}$ can be defined over the interval $[0,1]$ by

$$
U_{N}(\eta, t)=\sum_{j=m-1}^{m+2} \delta_{j} \phi_{j} .
$$

Using B-splines (5) and trial function (6), we can write the nodal values of $U, U^{\prime}, U^{\prime \prime}$ with respect to the time parameters $\delta_{m}$ in the following form:

$$
\begin{aligned}
& U_{m}=U\left(x_{m}\right)=\delta_{m-1}+4 \delta_{m}+\delta_{m+1} \\
& U_{m}^{\prime}=U^{\prime}\left(x_{m}\right)=3\left(-\delta_{m-1}+\delta_{m+1}\right) \\
& U_{m}^{\prime \prime}=U^{\prime \prime}\left(x_{m}\right)=6\left(\delta_{m-1}-2 \delta_{m}+\delta_{m+1}\right)
\end{aligned}
$$

where the superscript ' and " symbolize the first and second derivative to $\eta$, respectively. When applying the Galerkin's approach with weight function $W(x)$ to Eq.(1), we get the weak form of Eq.(1) as follows:

$$
\int_{a}^{b} W\left(U_{t}+\varepsilon U^{p} U_{x}-\mu U_{x x t} d x\right)=0 .
$$

Implementing the change of variable $x \rightarrow \eta$ to integral (8) yields

$$
\int_{0}^{1} W\left(U_{t}+\frac{\varepsilon}{h} \stackrel{\circ}{ }^{p} U_{\eta}-\frac{\mu}{h^{2}} U_{\eta \eta t}\right) d \eta=0,
$$

where $\stackrel{i}{U}$ is taken to be a constant over an element to simplify the integral. Applying partial integration once to (9) forms

$$
\int_{0}^{1}\left[W\left(U_{t}+\lambda U_{\eta}\right)+\beta W_{\eta} U_{\eta t}\right] d \eta=\left.\beta W U_{\eta t}\right|_{0} ^{1}
$$


where $\lambda=\frac{\varepsilon \dot{U}^{p}}{h}$ and $\beta=\frac{\mu}{h^{2}}$. Substituting cubic B-splines (5) instead of the weight function $W(x)$ and trial function (6) into integral equation (10) yield to the following form:

$$
\sum_{j=m-1}^{m+2}\left[\left(\int_{0}^{1} \phi_{i} \phi_{j}+\beta \phi_{i}^{\prime} \phi_{j}^{\prime}\right) d \eta-\left.\beta \phi_{i} \phi_{j}^{\prime}\right|_{0} ^{1}\right] \dot{\delta}_{j}^{e}+\sum_{j=m-1}^{m+2}\left(\lambda \int_{0}^{1} \phi_{i} \phi_{j}^{\prime} d \eta\right) \delta_{j}^{e}=0,
$$

in which $\delta^{e}=\left(\delta_{m-1}, \delta_{m}, \delta_{m+1}, \delta_{m+2}\right)^{T}$ and the dot states differentiation to $t$. This equation can be written in matrix form by

$$
\left[A^{e}+\beta\left(B^{e}-C^{e}\right)\right] \dot{\delta}^{e}+\lambda D^{e} \delta^{e}=0 .
$$

The element matrices are explained as follows:

$$
\begin{gathered}
A_{i j}^{e}=\int_{0}^{1} \phi_{i} \phi_{j} d \eta=\frac{1}{140}\left[\begin{array}{cccc}
20 & 129 & 60 & 1 \\
129 & 1188 & 933 & 60 \\
60 & 933 & 1188 & 129 \\
1 & 60 & 129 & 20
\end{array}\right] \\
B_{i j}^{e}=\int_{0}^{1} \phi_{i}^{\prime} \phi_{j}^{\prime} d \eta=\frac{1}{10}\left[\begin{array}{cccc}
18 & 21 & -36 & -3 \\
21 & 102 & -87 & -36 \\
-36 & -87 & 102 & 21 \\
-3 & -36 & 21 & 18
\end{array}\right] \\
C_{i j}^{e}=\left.\phi_{i} \phi_{j}^{\prime}\right|_{0} ^{1}=3\left[\begin{array}{cccc}
1 & 0 & -1 & 0 \\
4 & -1 & -4 & 1 \\
1 & -4 & -1 & 4 \\
0 & -1 & 0 & 1
\end{array}\right] \\
D_{i j}^{e}=\int_{0}^{1} \phi_{i} \phi_{j}^{\prime} d \eta=\frac{1}{20}\left[\begin{array}{cccc}
-10 & -9 & 18 & 1 \\
-71 & -150 & 183 & 38 \\
-38 & -183 & 150 & 71 \\
-1 & -18 & 9 & 10
\end{array}\right]
\end{gathered}
$$

with the subscript $i, j=m-1, m, m+1, m+2$. A lumped form of $\lambda$ calculated from $\left(\frac{U_{m}+U_{m+1}}{2}\right)^{p}$ is

$$
\lambda=\frac{\varepsilon}{2^{p} h}\left(\delta_{m-1}+5 \delta_{m}+5 \delta_{m+1}+\delta_{m+2}\right)^{p} .
$$

By considering together contributions from all elements, the matrix equation (12) becomes

$$
[A+\beta(B-C)] \dot{\delta}+\lambda D \delta=0,
$$

where $\delta=\left(\delta_{-1}, \delta_{0}, \ldots, \delta_{N}, \delta_{N+1}\right)^{T}$ is a nodal parameters. The $A, B$ and $\lambda D$ are septa-diagonal matrices and their line of $m$ is

$$
\begin{aligned}
& A=\frac{1}{140}(1,120,1191,2416,1191,120,1) \\
& B=\frac{1}{10}(-3,-72,-45,240,-45,-72,-3) \\
& \lambda D=\frac{1}{20}\left(\begin{array}{c}
-\lambda_{1},-18 \lambda_{1}-38 \lambda_{2}, 9 \lambda_{1}-183 \lambda_{2}-71 \lambda_{3}, 10 \lambda_{1}+150 \lambda_{2}-150 \lambda_{3}-10 \lambda_{4}, \\
71 \lambda_{2}+183 \lambda_{3}-9 \lambda_{4}, 38 \lambda_{3}+18 \lambda_{4}, \lambda_{4}
\end{array}\right)
\end{aligned}
$$

where

$$
\begin{aligned}
& \lambda_{1}=\frac{\varepsilon}{2^{p h}}\left(\delta_{m-2}+5 \delta_{m-1}+5 \delta_{m}+\delta_{m+1}\right)^{p}, \lambda_{2}=\frac{\varepsilon}{2^{p h}}\left(\delta_{m-1}+5 \delta_{m}+5 \delta_{m+1}+\delta_{m+2}\right)^{p}, \\
& \lambda_{3}=\frac{\varepsilon}{2^{p h}}\left(\delta_{m}+5 \delta_{m+1}+5 \delta_{m+2}+\delta_{m+3}\right)^{p}, \lambda_{4}=\frac{\varepsilon}{2^{p h}}\left(\delta_{m+1}+5 \delta_{m+2}+5 \delta_{m+3}+\delta_{m+4}\right)^{p} .
\end{aligned}
$$


Applying the forward finite difference $\dot{\delta}=\frac{\delta^{n+1}-\delta^{n}}{\Delta t}$ and Crank-Nicolson approach $\delta=\frac{1}{2}\left(\delta^{n}+\delta^{n+1}\right)$ to equation (13), we can easily achieve the septa-diagonal matrix system

$$
\left[A+\beta(B-C)+\frac{\lambda \Delta t}{2} D\right] \delta^{n+1}=\left[A+\beta(B-C)-\frac{\lambda \Delta t}{2} D\right] \delta^{n} .
$$

Using the boundary conditions given by (2), the $(N+3) \times(N+3)$ system $(14)$ is reduced to $(N+1) \times(N+1)$ septa-diagonal matrix system. This equation system can be solved by using Thomas algorithm. In this solution process, we need to two or three inner iterations $\delta^{n *}=\delta^{n}+\frac{1}{2}\left(\delta^{n}-\delta^{n-1}\right)$ at each time step to minimize the effect of non-linearity. Eventually, we obtain the recurrence relationship between two time steps $n$ and $n+1$ as an ordinary member of the matrix system (14)

$$
\begin{aligned}
& \gamma_{1} \delta_{m-3}^{n+1}+\gamma_{2} \delta_{m-2}^{n+1}+\gamma_{3} \delta_{m-1}^{n+1}+\gamma_{4} \delta_{m}^{n+1}+\gamma_{5} \delta_{m+1}^{n+1}+\gamma_{6} \delta_{m+2}^{n+1}+\gamma_{7} \delta_{m+3}^{n+1}= \\
& \gamma_{7} \delta_{m-3}^{n}+\gamma_{6} \delta_{m-2}^{n}+\gamma_{5} \delta_{m-1}^{n}+\gamma_{4} \delta_{m}^{n}+\gamma_{3} \delta_{m+1}^{n}+\gamma_{2} \delta_{m+2}^{n}+\gamma_{1} \delta_{m+3}^{n},
\end{aligned}
$$

where

$$
\begin{array}{ll}
\gamma_{1}=\frac{1}{140}-\frac{3 \beta}{10}-\frac{\lambda \Delta t}{40}, & \gamma_{2}=\frac{120}{140}-\frac{72 \beta}{10}-\frac{56 \lambda \Delta t}{40} \\
\gamma_{3}=\frac{1191}{140}-\frac{45 \beta}{10}-\frac{245 \lambda \Delta t}{40}, & \gamma_{4}=\frac{2416}{140}+\frac{240 \beta}{10}, \\
\gamma_{5}=\frac{1191}{140}-\frac{45 \beta}{10}+\frac{245 \lambda \Delta t}{40}, & \gamma_{6}=\frac{120}{140}-\frac{72 \beta}{10}+\frac{56 \lambda \Delta t}{40} \\
\gamma_{7}=\frac{1}{140}-\frac{3 \beta}{10}+\frac{\lambda \Delta t}{40} . &
\end{array}
$$

In order to start the iteration, the initial vector $\delta^{0}$ must be computed by using the initial and boundary conditions. Because of this, using the relations at the knots $U_{N}\left(x_{m}, 0\right)=U\left(x_{m}, 0\right), \quad m=0,1, \cdots, N$ and derivative condition $U_{N}^{\prime}\left(x_{0}, 0\right)=U^{\prime}\left(x_{N}, 0\right)=0$ together with a variant of the Thomas algorithm, the initial vector $\delta^{0}$ can be easily calculated from the following matrix form

$$
\left[\begin{array}{ccccccc}
-3 & 0 & 3 & & & & \\
1 & 4 & 1 & & & & \\
& & & \ddots & & & \\
& & & & 1 & 4 & 1 \\
& & & & -3 & 0 & 3
\end{array}\right]\left[\begin{array}{c}
\delta_{-1}^{0} 1 \\
\delta_{0}^{0} \\
\vdots \\
\delta_{N}^{0} \\
\delta_{N+1}^{0}
\end{array}\right]=\left[\begin{array}{c}
U^{\prime}\left(x_{0}, 0\right) \\
U\left(x_{0}, 0\right) \\
\vdots \\
U\left(x_{N}, 0\right) \\
U^{\prime}\left(x_{N}, 0\right)
\end{array}\right]
$$

\subsection{Stability analysis}

For the linear stability analysis of the numerical algorithm, we use the Fourier method and assume that the quantity $U^{p}$ in the non-linear term $U^{p} U_{x}$ of GEW equation is locally constant. Substituting the Fourier mode $\delta_{j}^{n}=g^{n} e^{i j k h}$ where $k$ is mode number and $h$ is the element size, into the scheme (15), this leads to the growth factor

$$
g=\frac{a-i b}{a+i b},
$$

where

$$
\begin{aligned}
& a=\left(\gamma_{7}+\gamma_{1}\right) \cos (3 k h)+\left(\gamma_{6}+\gamma_{2}\right) \cos (2 k h)+\left(\gamma_{5}+\gamma_{3}\right) \cos (k h)+\gamma_{4}, \\
& b=\left(\gamma_{7}-\gamma_{1}\right) \sin (3 k h)+\left(\gamma_{6}-\gamma_{2}\right) \sin (2 k h)+\left(\gamma_{5}-\gamma_{3}\right) \sin (k h) .
\end{aligned}
$$

The modulus of $|g|$ is 1 which means that the scheme is unconditionally stable.

\section{Numerical Examples and Results}

The GEW equation has the solitary wave solution $[6,7,8]$

$$
U(x, t)=\sqrt[p]{\frac{c(p+1)(p+2)}{2 \varepsilon} \sec h^{2}\left[\frac{p}{2 \sqrt{\mu}}\left(x-c t-x_{0}\right)\right]}
$$


where $c$ is the the constant velocity of the wave traveling in the positive direction of the $x$-axis, $x_{0}$ is arbitrary constant. Moreover, the equation possesses the three conservation laws

$$
I_{1}=\int_{a}^{b} U d x, \quad I_{2}=\int_{a}^{b}\left[U^{2}+\mu U_{x}^{2}\right] d x, \quad I_{3}=\int_{a}^{b} U^{p+2} d x
$$

which correspond to mass, momentum and energy. The numerical algorithm is tested with single solitary wave and interaction of two solitary wave problems. In these two problems, to measure the performance of the numerical method, the $L_{2}$ and $L_{\infty}$ error norms are computed by using the solitary wave solution in (18) and the following equalities:

$$
\begin{gathered}
L_{2}=\left\|U^{\text {exact }}-U_{N}\right\|_{2} \simeq \sqrt{h \sum_{J=0}^{N}\left|U_{j}^{\text {exact }}-\left(U_{N}\right)_{j}\right|^{2}}, \\
L_{\infty}=\left\|U^{\text {exact }}-U_{N}\right\|_{\infty} \simeq \max _{j}\left|U_{j}^{\text {exact }}-\left(U_{N}\right)_{j}\right| .
\end{gathered}
$$

The changes of the invariants (19) are also observed to indicate the conservation properties of the numerical approach.

\subsection{The Motion of Single Solitary Wave}

For this problem, the five sets of parameters by taking different values of $p, c$ and amplitude $=\sqrt[p]{\frac{c(p+1)(p+2)}{2 \varepsilon}}$ and the same values of $h=0.1, \Delta t=0.2, \varepsilon=3, \mu=1, x_{0}=30,0 \leq x \leq 80$ is considered to coincide with papers $[6,7,8]$. The numerical simulations are run from the time $t=0$ to time $t=20$.

In the first case, we choose the quantities $p=2, c=1 / 32$ and $1 / 2$. Hence, the solitary wave has amplitude $=$ 0.25 and 1 , respectively. The calculated quantities of the invariants are presented in Tables I, II. As can be seen in Table I, three invariants are almost constant as the time increases. Table II shows that the changes of the invariants from their initial state are less than $2 \%, 3 \%$ and $3 \%$, respectively. Also, we have found out that the quantity of the error norms $L_{2}$ and $L_{\infty}$ is reasonably small, as expected.

Table I. The invariants and the error norms for single solitary wave with $p=2$, amplitude $=0.25, \Delta t=0.2, h=0.1, \varepsilon=$ $3, \mu=1,0 \leq x \leq 80$.

\begin{tabular}{cccccc}
\hline \hline Time & $I_{1}$ & $I_{2}$ & $I_{3}$ & $L_{2} \times 10^{5}$ & $L_{\infty} \times 10^{5}$ \\
\hline 0 & 0.7853966 & 0.1666661 & 0.0052083 & 0.00000000 & 0.00000000 \\
5 & 0.7853966 & 0.1666662 & 0.0052083 & 2.00511050 & 1.10880256 \\
10 & 0.7853967 & 0.1666662 & 0.0052083 & 3.99064595 & 2.22513057 \\
15 & 0.7853967 & 0.1666662 & 0.0052083 & 5.93886201 & 3.34204308 \\
20 & 0.7853968 & 0.1666663 & 0.0052083 & 7.83378959 & 4.44850332 \\
\hline \hline
\end{tabular}

Table II. The invariants and the error norms for single solitary wave with $p=2$, amplitude $=1, \Delta t=0.2, h=0.1, \varepsilon=$ $3, \mu=1,0 \leq x \leq 80$.

\begin{tabular}{cccccc}
\hline \hline Time & $I_{1}$ & $I_{2}$ & $I_{3}$ & $L_{2}$ & $L_{\infty}$ \\
\hline 0 & 3.1415863 & 2.6666583 & 1.3333283 & 0.00000000 & 0.00000000 \\
5 & 3.1458905 & 2.6724969 & 1.3391718 & 0.01650411 & 0.01116168 \\
10 & 3.1502060 & 2.6783543 & 1.3450481 & 0.02812323 & 0.01888483 \\
15 & 3.1545617 & 2.6842736 & 1.3509996 & 0.03526213 & 0.02393891 \\
20 & 3.1589605 & 2.6902580 & 1.3570299 & 0.03803037 & 0.02629007 \\
\hline \hline
\end{tabular}


Secondly, if $p=3, c=0.001$ and $c=0.3$, the solitary wave has amplitude $=0.15$ and 1 . The obtained results are given in Tables III, IV. It is observed from Table III that three invariants are nearly unchanged as the time processes. In Table IV, the changes of the invariants are less than $2 \%, 3 \%$ and $3 \%$, respectively. In addition, The values of the error norms $L_{2}$ and $L_{\infty}$ are adequately small.

Table III. The invariants and the error norms for single solitary wave with $p=3$, amplitude $=0.15, \Delta t=0.2, h=0.1, \varepsilon=$ $3, \mu=1,0 \leq x \leq 80$.

\begin{tabular}{cccccc}
\hline \hline Time & $I_{1}$ & $I_{2}$ & $I_{3}$ & $L_{2} \times 10^{7}$ & $L_{\infty} \times 10^{7}$ \\
\hline 0 & 0.4189154 & 0.0549805 & 0.0000733 & 0.00000000 & 0.00000000 \\
5 & 0.4189154 & 0.0549805 & 0.0000733 & 7.06255100 & 4.57700375 \\
10 & 0.4189154 & 0.0549805 & 0.0000733 & 14.12494884 & 9.16012912 \\
15 & 0.4189154 & 0.0549805 & 0.0000733 & 21.18707557 & 13.74545441 \\
20 & 0.4189154 & 0.0549805 & 0.0000733 & 28.24881328 & 18.32910252 \\
\hline \hline
\end{tabular}

Table IV. The invariants and the error norms for single solitary wave with $p=3$, amplitude $=1, \Delta t=0.2, h=0.1, \varepsilon=$ $3, \mu=1,0 \leq x \leq 80$.

\begin{tabular}{cccccc}
\hline \hline Time & $I_{1}$ & $I_{2}$ & $I_{3}$ & $L_{2}$ & $L_{\infty}$ \\
\hline 0 & 2.8043580 & 2.4639009 & 0.9855618 & 0.00000000 & 0.00000000 \\
5 & 2.8079094 & 2.4691674 & 0.9908334 & 0.01048354 & 0.00825708 \\
10 & 2.8114726 & 2.4744442 & 0.9961351 & 0.01657542 & 0.01277205 \\
15 & 2.8150809 & 2.4797940 & 1.0015278 & 0.01850263 & 0.01460246 \\
20 & 2.8187398 & 2.4852249 & 1.0070200 & 0.01655637 & 0.01370453 \\
\hline \hline
\end{tabular}

Finally, we take the parameters $p=4, c=0.2$. This leads to amplitude $=1$. The obtained results are listed in Table $\mathrm{V}$ which clearly shows that the change of the invariants from their initial count are less than $2 \%$. Also, we observed that the quantity of the error norms $L_{2}$ and $L_{\infty}$ is sensibly small.

Table $\mathrm{V}$. The invariants and the error norms for single solitary wave with $p=4$, amplitude $=1, \Delta t=0.2, h=0.1, \varepsilon=$ $3, \mu=1,0 \leq x \leq 80$.

\begin{tabular}{cccccc}
\hline \hline Time & $I_{1}$ & $I_{2}$ & $I_{3}$ & $L_{2}$ & $L_{\infty}$ \\
\hline 0 & 2.6220516 & 2.3561722 & 0.7853952 & 0.00000000 & 0.00000000 \\
5 & 2.6247173 & 2.3603671 & 0.7895906 & 0.00691120 & 0.00632109 \\
10 & 2.6273729 & 2.3645238 & 0.7937683 & 0.01055235 & 0.00914085 \\
15 & 2.6300579 & 2.3687299 & 0.7980116 & 0.01104764 & 0.00976358 \\
20 & 2.6327833 & 2.3730032 & 0.8023383 & 0.00890617 & 0.00821991 \\
\hline \hline
\end{tabular}

The motion of a single solitary wave is plotted at different time levels $t=0,10,20$ in Fig. 1, 2. It is understood from these figures that the numerical scheme performs the motion of propagation of a single solitary wave, which moves to the right at nearly unchanged speed and conserves its amplitude and shape with increasing time.

The comparison of our results with the ones obtained by collocation methods based on quadratic, cubic B-spline $[6,7]$ and Petrov-Galerkin method [8] at $t=20$ is given in Table VI. From this table, we can conclude that the values of three invariants are to be close to each other. The magnitude of our error norms is smaller than the ones given by [6, 7] for $p=2,3$ and it is almost same with the paper [8] for $p=4$. 


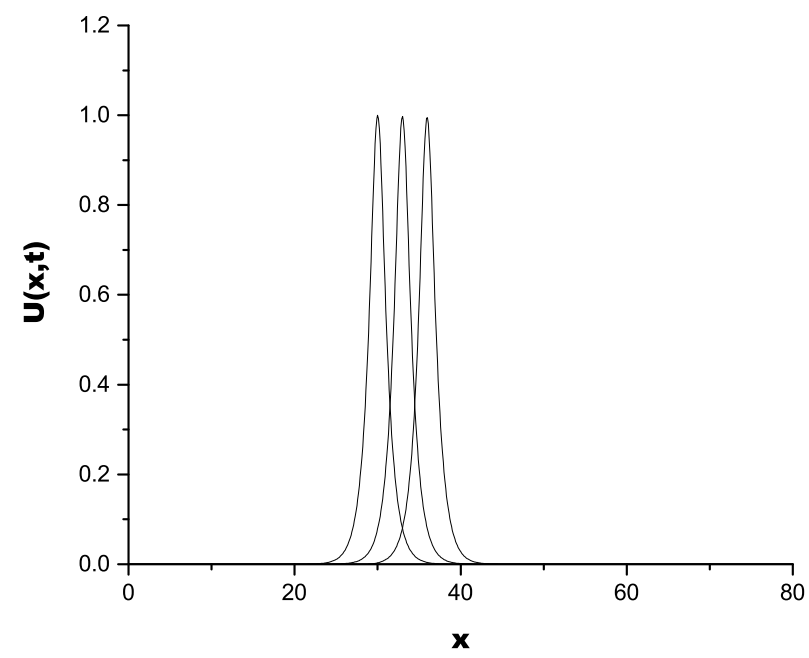

Figure 1. Single solitary wave with $p=3, c=0.3, x_{0}=30,0 \leq x \leq 80, t=0,10,20$.

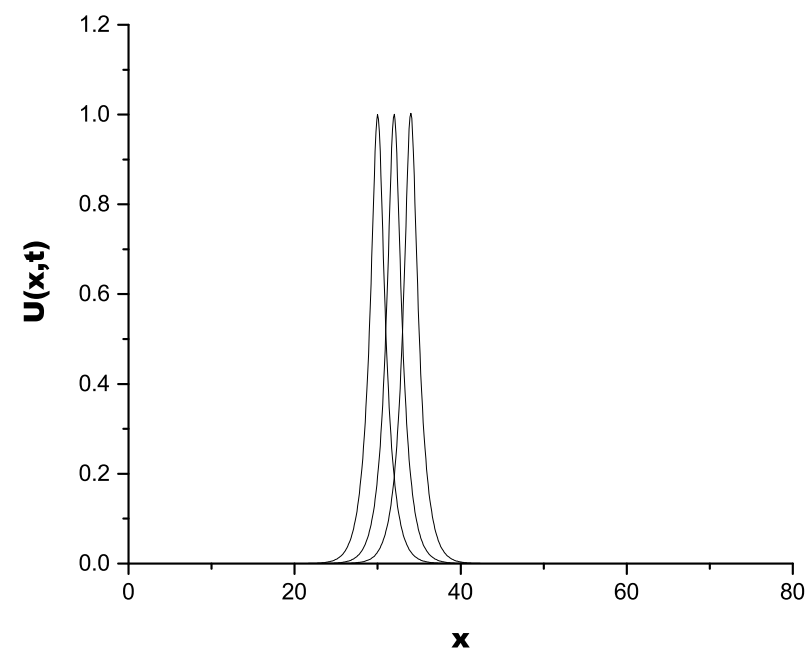

Figure 2. Single solitary wave with $p=4, c=0.2, x_{0}=30,0 \leq x \leq 80, t=0,10,20$.

\subsection{The Interaction of Two Solitary Waves}

In the second test problem, we use the initial condition

$$
U(x, 0)=\sum_{i=1}^{2} \sqrt[p]{\frac{c_{i}(p+1)(p+2)}{2 \varepsilon} \sec h^{2}\left[\frac{p}{2 \sqrt{\delta}}\left(x-x_{i}\right)\right]},
$$

which produces two positive solitary waves having different amplitudes of magnitudes 1 and 0.5 at the same direction, where $c_{i}$ and $x_{i}, i=1,2$ are arbitrary constants. 
Table VI. For $p=2,3$ and 4 , Comprasions of result for the single solitary wave with $\Delta t=0.2, h=0.1, \varepsilon=3, \mu=1$, $0 \leq x \leq 80$.

\begin{tabular}{ccccc}
\hline & $p$ & 2 & 3 & 4 \\
\hline \multirow{4}{*}{$I_{1}$} & Collocation (quadratic)[6] & 0.78528640 & & \\
& Collocation (cubic)[7] & 0.78466760 & 0.65908330 & \\
& Petrov-Galerkin (quadratic)[8] & 0.78539800 & 0.41891600 & 2.62206000 \\
& Ours - Galerkin (cubic) & 0.78539680 & 0.41891540 & 2.63278330 \\
\hline \multirow{4}{*}{$I_{2}$} & Collocation (quadratic)[6] & 0.16658180 & & \\
& Collocation (cubic)[7] & 0.16643400 & 0.05938137 & \\
& Petrov-Galerkin (quadratic)[8] & 0.16666900 & 0.05497830 & 2.35615000 \\
& Ours - Galerkin (cubic) & 0.16666630 & 0.05498050 & 2.37300320 \\
\hline \multirow{4}{*}{$I_{3}$} & Collocation (quadratic)[6] & 0.00520600 & & \\
& Collocation (cubic)[7] & 0.00519380 & 0.00006871 & \\
& Petrov-Galerkin (quadratic)[8] & 0.00520829 & 0.00007330 & 0.78534400 \\
& Ours - Galerkin (cubic) & 0.00520830 & 0.00007330 & 0.8023383 \\
\hline \multirow{4}{*}{$L_{2} \times 10^{3}$} & Collocation (quadratic)[6] & 0.15695390 & & \\
& Collocation (cubic)[7] & 0.19588780 & 0.51496770 & \\
& Petrov-Galerkin (quadratic)[8] & 0.00250172 & 0.00006407 & 2.30499000 \\
& Ours - Galerkin (cubic) & 0.07833789 & 0.00282488 & 8.90617000 \\
\hline \multirow{4}{*}{$L_{\infty} \times 10^{3}$} & Collocation (quadratic)[6] & 0.20214760 & & \\
& Collocation (cubic)[7] & 0.17443300 & 0.32060590 & \\
& Petrov-Galerkin (quadratic)[8] & 0.00275164 & 0.00008206 & 1.88285000 \\
& Ours - Galerkin (cubic) & 0.04448503 & 0.00183291 & 8.21991000 \\
\hline
\end{tabular}
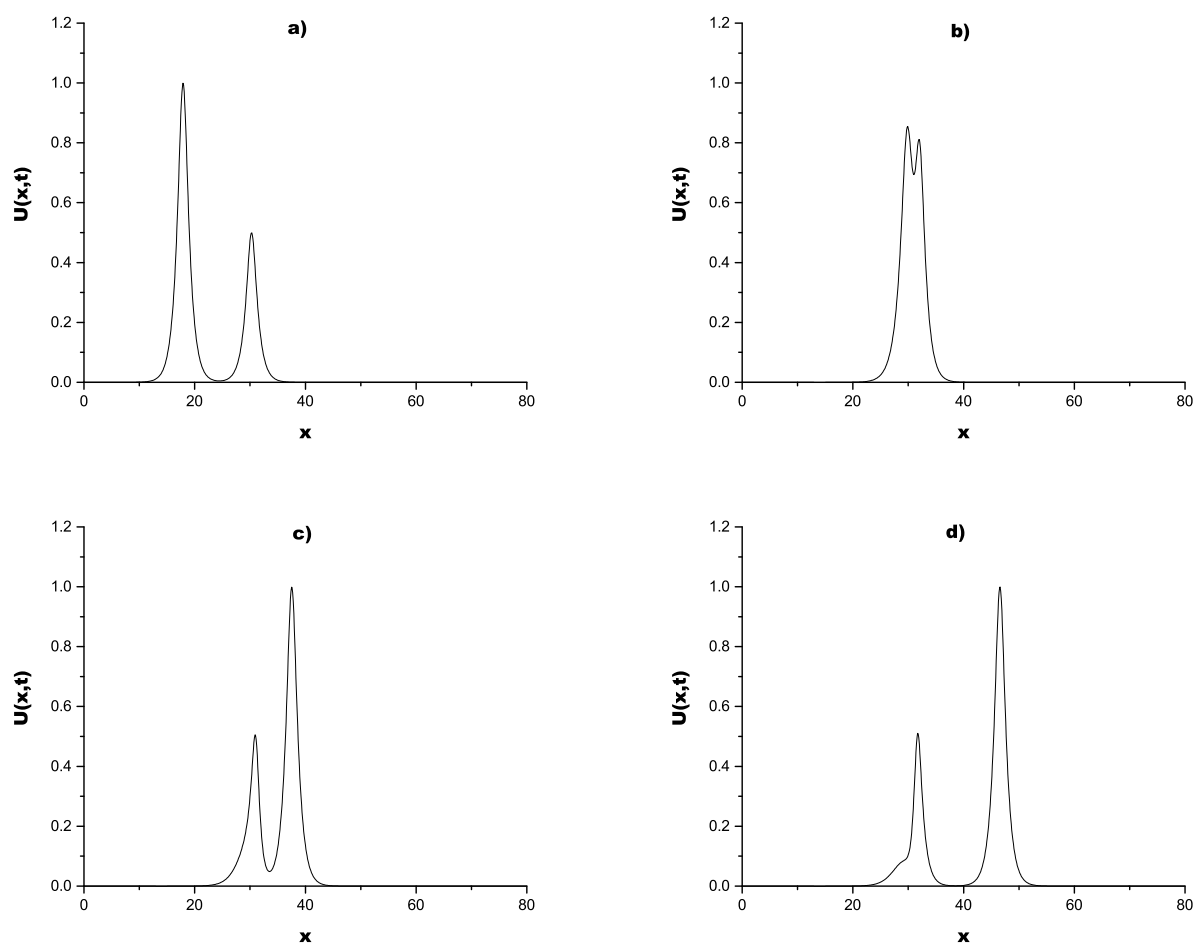

Figure 3. Interaction of two solitary waves at $p=3$; a) $t=10, b) t=50, c) t=70, d) t=100$. 

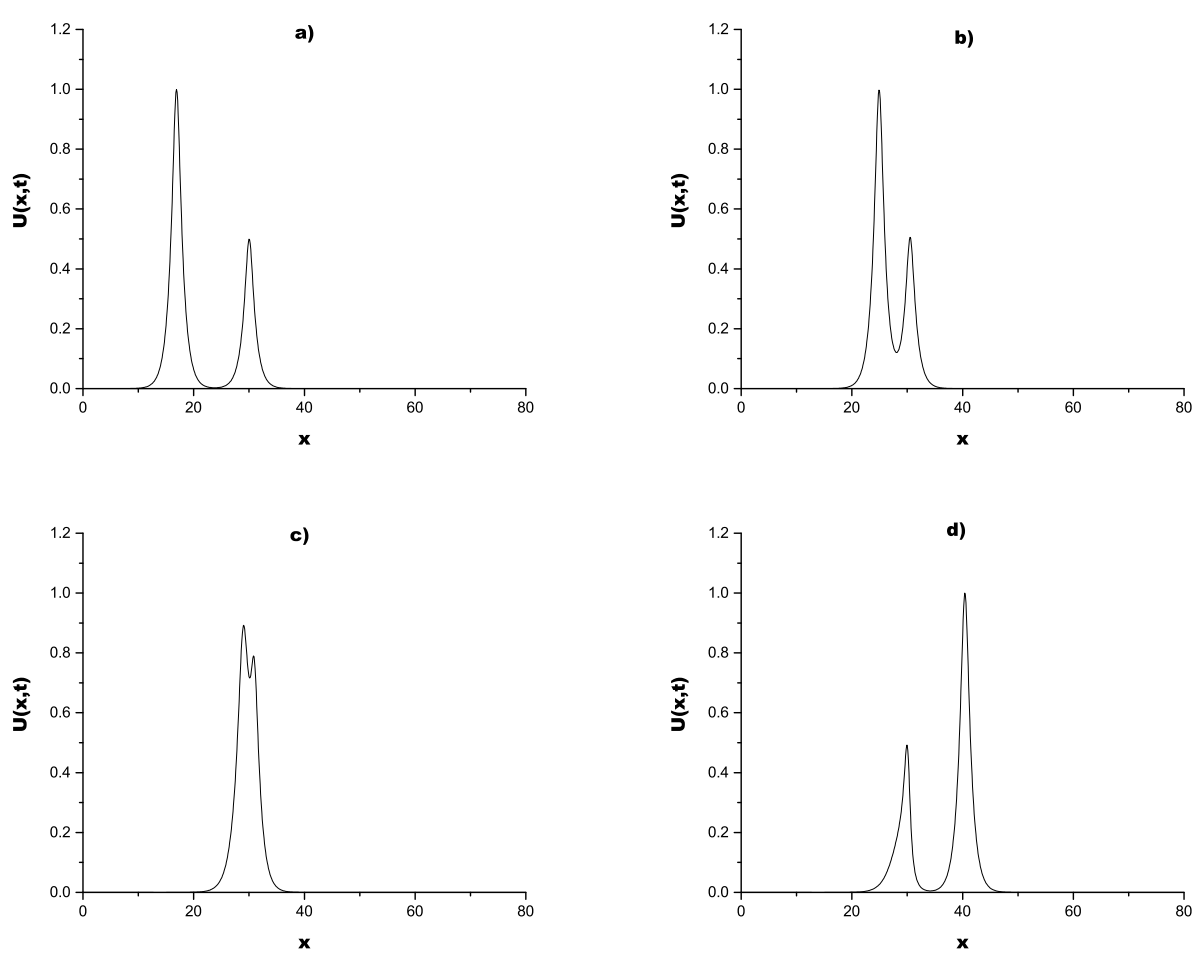

Figure 4. Interaction of two solitary waves at $p=4 ; a) t=10, b) t=50, c) t=70, d) t=120$.

Three sets of parameters have been constructed by taking the values of first parameters $p=2, c_{1}=0.5$ and $c_{2}=0.125$, second parameters $p=3, c_{1}=0.3$ and $c_{2}=0.0375$ and third parameters $p=4, c_{1}=0.2$ and $c_{2}=1 / 80$. The computer program is run until time $t=60,100$ and 120, respectively. The other parameters are considered as $h=0.1, \Delta t=0.025, \varepsilon=3, \mu=1, x_{1}=15, x_{2}=30,0 \leq x \leq 80$ to coincide with papers [6, 7, 8].

To prove the conservate quantities of the invariants $I_{1}, I_{2}$ and $I_{3}$, the calculated values are given in Table VII, VIII, IX which show that the change of the invariants from their initial case are less than $0.1 \%, 0.5 \%$ and $0.8 \%$. In addition, the invariant quantities are compatible with those of Roshan [8].

Table VII. The invariants for interaction of two solitary waves with $p=2, c_{1}=0.5, c_{2}=0.125, x_{1}=15, x_{2}=30, \Delta t=$ $0.025, h=0.1, \varepsilon=3, \mu=1,0 \leq x \leq 80$.

\begin{tabular}{|c|c|c|c|c|c|c|}
\hline \multirow[b]{2}{*}{ Time } & \multicolumn{2}{|c|}{$I_{1}$} & \multicolumn{2}{|c|}{$\overline{I_{2}}$} & \multicolumn{2}{|c|}{$\overline{I_{3}}$} \\
\hline & Ours-Galerkin & Pet.-Gal.[8] & Ours-Galerkin & Pet.-Gal.[8] & Ours-Galerkin & Pet.-Gal.[8] \\
\hline 0 & 4.71237 & 4.71239 & 3.33332 & 3.33324 & 1.41666 & 1.14166 \\
\hline 10 & 4.71236 & 4.71239 & 3.33331 & 3.33324 & 1.41665 & 1.14166 \\
\hline 20 & 4.71235 & 4.71239 & 3.33332 & 3.33324 & 1.41666 & 1.14166 \\
\hline 30 & 4.71260 & 4.71239 & 3.33416 & 3.33324 & 1.41758 & 1.14166 \\
\hline 40 & 4.71234 & 4.71239 & 3.33345 & 3.33333 & 1.41699 & 1.14166 \\
\hline 50 & 4.71210 & 4.71239 & 3.33290 & 3.33338 & 1.41652 & 1.14166 \\
\hline 60 & 4.71213 & 4.71239 & 3.33296 & 3.33333 & 1.41651 & 1.14166 \\
\hline
\end{tabular}

The interaction of two solitary waves is depicted at different time levels in Fig. 3, 4(a)-(d). In these figures, initially, the wave with larger amplitude is on the left of the second wave with smaller amplitude. In the progress of 
Table VIII. The invariants for interaction of two solitary waves with $p=3, c_{1}=0.3, c_{2}=0.0375, x_{1}=15, x_{2}=30, \Delta t=$ $0.025, h=0.1, \varepsilon=3, \mu=1,0 \leq x \leq 80$.

\begin{tabular}{|c|c|c|c|c|c|c|}
\hline \multirow[b]{2}{*}{ Time } & \multicolumn{2}{|c|}{$\bar{I} I_{1}$} & \multicolumn{2}{|c|}{$\overline{I_{2}}$} & \multicolumn{2}{|c|}{$I_{3}$} \\
\hline & Ours-Galerkin & Pet.-Gal.[8] & Ours-Galerkin & Pet.-Gal.[8] & Ours-Galerkin & Pet.-Gal.[8] \\
\hline 0 & 4.20653 & 4.20655 & 3.07987 & 3.97977 & 1.01636 & 1.01634 \\
\hline 10 & 4.20653 & 4.20655 & 3.07989 & 2.07986 & 1.01637 & 1.01634 \\
\hline 20 & 4.20652 & 4.20655 & 3.07988 & 3.07982 & 1.01635 & 1.01634 \\
\hline 30 & 4.20653 & 4.20655 & 3.07991 & 3.07980 & 1.01638 & 1.01634 \\
\hline 40 & 4.20677 & 4.20655 & 3.08050 & 3.07986 & 1.01698 & 1.01634 \\
\hline 50 & 4.20793 & 4.20655 & 3.08362 & 3.07981 & 1.02059 & 1.01633 \\
\hline 60 & 4.20616 & 4.20655 & 3.07947 & 3.07987 & 1.01654 & 1.01633 \\
\hline 70 & 4.20558 & 4.20655 & 3.07863 & 3.07976 & 1.01629 & 1.01634 \\
\hline 80 & 4.20509 & 4.20655 & 3.07800 & 3.07991 & 1.01620 & 1.01633 \\
\hline 90 & 4.20490 & 4.20655 & 3.07777 & 3.07974 & 1.01616 & 1.01633 \\
\hline 100 & 4.20503 & 4.20655 & 3.07797 & 3.07972 & 1.01616 & 1.01634 \\
\hline
\end{tabular}

Table IX. The invariants for interaction of two solitary waves with $p=4, c_{1}=0.2, c_{2}=1 / 80, x_{1}=15, x_{2}=30, \Delta t=$ $0.025, h=0.1, \varepsilon=3, \mu=1,0 \leq x \leq 80$.

\begin{tabular}{|c|c|c|c|c|c|c|}
\hline \multirow[b]{2}{*}{ Time } & \multicolumn{2}{|c|}{$\overline{I_{1}}$} & \multicolumn{2}{|c|}{$I_{2}$} & \multicolumn{2}{|c|}{$\overline{I_{3}}$} \\
\hline & Ours-Galerkin & Pet.-Gal.[8] & Ours-Galerkin & Pet.-Gal.[8] & Ours-Galerkin & Pet.-Gal.[8] \\
\hline 0 & 3.93307 & 3.93309 & 2.94521 & 2.94512 & 0.79766 & 0.79761 \\
\hline 10 & 3.93310 & 3.93309 & 2.94529 & 2.94518 & 0.79773 & 0.79761 \\
\hline 20 & 3.93309 & 3.93309 & 2.94527 & 2.94517 & 0.79771 & 0.79761 \\
\hline 30 & 3.93309 & 3.93309 & 2.94527 & 2.94510 & 0.79770 & 0.79761 \\
\hline 40 & 3.93310 & 3.93309 & 2.94529 & 2.94515 & 0.79773 & 0.79761 \\
\hline 50 & 3.93320 & 3.93309 & 2.94553 & 2.94504 & 0.79795 & 0.79761 \\
\hline 60 & 3.93388 & 3.93309 & 2.94703 & 2.94505 & 0.79942 & 0.79762 \\
\hline 70 & 3.93601 & 3.93307 & 2.95212 & 2.94510 & 0.80505 & 0.79763 \\
\hline 80 & 3.93285 & 3.93309 & 2.94529 & 2.94506 & 0.79862 & 0.79761 \\
\hline 90 & 3.93222 & 3.93309 & 2.94436 & 2.94520 & 0.79812 & 0.79761 \\
\hline 100 & 3.93161 & 3.93309 & 2.94366 & 2.94508 & 0.79805 & 0.79761 \\
\hline 110 & 3.93095 & 3.93309 & 2.94291 & 2.94517 & 0.79799 & 0.79761 \\
\hline 120 & 3.93026 & 3.93308 & 2.94212 & 2.94511 & 0.79794 & 0.79761 \\
\hline
\end{tabular}

time, the large wave catches up with the smaller one and overlapping process occurs. In time, waves start to resume their original shapes.

\section{Conclusion}

In this paper, we have obtained the solitary-wave solutions of the GEW equation using lumped Galerkin method based on cubic B-spline functions. To prove the performance of numerical scheme, the error norms $L_{2}$ and $L_{\infty}$ for single solitary wave and three invariants $I_{1}, I_{2}$ and $I_{3}$ for two test problems have been calculated. These calculations represent that our error norms are adequately small and they are smaller than or too close to the ones in existing numerical results. The changes of the invariants are sufficiently small and the quantities of the invariants are consistent with those of Roshan. Also, the linearized numerical scheme is unconditionally stable. Finally, we can say that our numerical method can be reliably used to obtain the numerical solution of the GEW equation and similar type non-linear equations. 


\section{REFERENCES}

1. D. H. Peregrine, Long waves on a beach, Journal of Fluid Mechanics, vol. 27, no. 4, pp. 815-827, 1967.

2. T. B. Benjamin, J. L. Bona and J. J. Mahony, Model equations for long waves in non-linear dispersive systems, Philosophical Transactions of the Royal Society of London Series A, vol. 272, pp. 47-78, 1972.

3. P. J. Morrison, J. D. Meiss and J. R. Carey, Scattering of RLW solitary waves, Physica D, vol. 11, pp. 324-336, 1984.

4. P. M. Prenter, Splines and variational methods, J. Wiley, New York, 1975.

5. S. Hamdi, W. H. Enright, W. E. Schiesser, J. J. Gottlieb and A. Alaal, Exact solutions of the generalized equal width wave equation, in: Proceedings of the International Conference on Computational Science and Its Applications, LNCS, vol. 2668, pp. 725-734, 2003.

6. D. J. Evans and K. R. Raslan, Solitary waves for the generalized equal width (GEW) equation, International Journal of Computer Mathematics, vol. 82, no. 4, pp. 445-455, 2005.

7. K. R. Raslan, Collocation method using cubic B-spline for the generalised equal width equation, Int. J. Simulation and Process Modelling, vol. 2, pp. 37-44, 2006.

8. T. Roshan, A Petrov-Galerkin method for solving the generalized equal width (GEW) equation, Journal of Computational and Applied Mathematics, vol. 235, pp. 1641-1652, 2011.

9. H. Panahipour, Numerical simulation of GEW equation using RBF collocation method, Communications in Numerical Analysis, vol. 2012, pp. 1-28, 2012.

10. N. Taghizadeh, M. Mirzazadeh, M. Akbari and M. Rahimian, Exact solutions for generalized equal width equation, Math. Sci. Lett. 2, vol. 2, pp. 99-106, 2013.

11. L. R. T. Gardner and G. A. Gardner, Solitary waves of the equal width wave equation, Journal of Computational Physics, vol. 101, no. 1, pp. 218-223, 1991.

12. L. R. T. Gardner, G. A. Gardner, F. A. Ayoup and N. K. Amein, Simulations of the EW undular bore, Commun. Numer. Meth. En., vol. 13, no. 7, pp. 583-592, 1997.

13. S. I. Zaki, A least-squares finite element scheme for the EW equation, Comp. Methods in Appl. Mech. and Eng., vol. 189, no. 2, pp. 587-594, 2000.

14. A. Doğan, Application of Galerkin's method to equal width wave equation, Applied Mathematics and Computation, vol. 160, pp. 65-76, 2005.

15. A. Esen, A numerical solution of the equal width wave equation by a lumped Galerkin method, Applied Mathematics and Computation, vol. 168, no. 1, pp. 270-282, 2005.

16. B. Saka, A finite element method for equal width equation, Applied Mathematics and Computation, vol. 175, no. 1, pp. 730-747, 2006.

17. A. Esen, A lumped Galerkin method for the numerical solution of the modified equal-width wave equation using quadratic B-splines, International Journal of Computer Mathematics, vol. 83, no. 5-6, pp. 449-459, 2006.

18. B. Saka, Algorithms for numerical solution of the modified equal width wave equation using collocation method, Mathematical and Computer Modelling, vol. 45, no. 9-10, pp. 1096-1117, 2007.

19. S. B. G. Karakoç and T. Geyikli, Numerical solution of the modified equal width wave equation, International Journal of Differential Equations, vol. 2012, pp. 1-15, 2012.

20. T. Geyikli and S. B. G. Karakoç, Petrov-Galerkin method with cubic B-splines for solving the MEW equation, Bull. Belg. Math. Soc. Simon Stevin, vol. 19, pp. 215-227, 2012.

21. L. R. T. Gardner and G. A. Gardner, Solitary waves of the regularized long wave equation, J. Comput. Phys., vol. 91, pp. 441-459, 1990.

22. A. Doğan, Numerical solution of RLW equation using linear finite elements within Galerkin's method, Applied Mathematical Modelling, vol. 26, pp. 771-783, 2002.

23. İ. Dağ, B. Saka and D. Irk, Galerkin method for the numerical solution of the RLW equation using quintic B-splines, Journal of Computational and Applied Mathematics, vol. 190, pp. 532-547, 2006.

24. B. Saka and İ. Dağ, Quartic B-spline Galerkin approach to the numerical solution of the KdVB equation, Applied Mathematics and Computation, vol. 215, no. 2, pp. 746-758, 2009.

25. S. Kutluay and Y. Uçar, A Quadratic B-spline Galerkin Approach for Solving a Coupled KdV Equation, Mathematical Modelling and Analysis, vol. 18, no. 1, pp. 103-121, 2013

26. S. B. G. Karakoç, Y. Uçar and N. Yağmurlu, Numerical solutions of the MRLW equation by cubic B-spline Galerkin finite element method, Kuwait Journal of Science, vol. 42, no. 2, pp. 141-159, 2015.

27. S. B. G. Karakoç, H. Zeybek and T. Ak, Numerical solutions of the Kawahara equation by the septic B-spline collocation method, Statistics, Optimization \& Information Computing, vol. 2, no. 3, pp. 211-221, 2014.

28. A. Esen, Y. Uçar, N. Yağmurlu and O. Tasbozan, A Galerkin Finite Element Method to Solve Fractional Diffusion and Fractional Diffusion-Wave Equations, Mathematical Modelling and Analysis, vol. 18, no. 2, pp. 260-273, 2013.

29. Y. Uçar, B. Karaagac and A. Esen, A new approach on numerical solutions of the Improved Boussinesq type equation using quadratic B-spline Galerkin finite element method, Applied Mathematics and Computation, vol. 270, pp. 148-155, 2015.

30. S. Kutluay and Y. Uçar, Numerical solution of a coupled modified Kortewegde Vries equation by the Galerkin method using quadratic $B$-splines, International Journal of Computer Mathematics, vol. 90, no. 11, pp. 2353-2371, 2013. 